\title{
Je suis un intrus en criminologie : une conversation avec Pieter Spierenburg
}

Entretien conduit par Tom Daems et René van Swaaningen

Pieter Spierenburg, Tom Daems et René van Swaaningen

\section{(2) OpenEdition}

\section{Journals}

Édition électronique

URL : https://journals.openedition.org/chs/2569

DOI : $10.4000 /$ chs. 2569

ISSN : 1663-4837

\section{Éditeur}

Librairie Droz

\section{Édition imprimée}

Date de publication : 18 décembre 2019

Pagination : 107-121

ISSN : 1422-0857

\section{Référence électronique}

Pieter Spierenburg, Tom Daems et René van Swaaningen, « Je suis un intrus en criminologie : une conversation avec Pieter Spierenburg », Crime, Histoire \& Sociétés / Crime, History \& Societies [En ligne], vol. 23, n² | 2019, mis en ligne le 06 mai 2020, consulté le 24 novembre 2022. URL : http:// journals.openedition.org/chs/2569; DOI : https://doi.org/10.4000/chs.2569

Ce document a été généré automatiquement le 24 novembre 2022.

Tous droits réservés 


\title{
Je suis un intrus en criminologie : une conversation avec Pieter Spierenburg
}

\author{
Entretien conduit par Tom Daems et René van Swaaningen
}

Pieter Spierenburg, Tom Daems et René van Swaaningen

\section{NOTE DE L'AUTEUR}

Texte original : Daems, T., van Swaaningen, R., Ik ben een beunhaas in de criminologie, Tijdschrift over Cultuur \& Criminaliteit, 2019, 9, 1, 16-30. Traduction française :

Xavier Rousseaux avec l'aide de René Lévy et Margo De Koster que je remercie pour leurs suggestions. Merci également à Tom Daens et René van Swaaningen pour nous avoir autorisés à traduire le texte original. Les notes du traducteur destinées à familiariser les lecteurs avec le contexte intellectuel néerlandais sont de ma responsabilité (Xavier Rousseaux).

Nous avons rencontré Pieter Spierenburg le 2 novembre 2018 dans sa maison, un bâtiment
majestueux du quartier du Nieuwe markt (nouveau marché) d'Amsterdam, situé sur l'eau, à
deux pas de la gare centrale. Dans le salon se trouve une belle gravure de la maison de
correction la plus célèbre du monde, la Rasphuis d'Amsterdam, qui ouvrit ses portes en
1596 ; ce n'est bien sûr pas un hasard, étant donné son expertise historique et
criminologique. Aujourd'hui, la porte restaurée de la défunte Rasphuis sur le Heiligeweg
donne accès à un centre commercial. Une plaque de bronze avec des explications concises
à l'intention des passants et des touristes et le mot castigatio (châtiment) rappellent le dur
labeur qui a été fait ici pour râper le bois brésilien comme matière première pour la peinture.
La discipline et l'éthique du travail cèdent aujourd'hui la place au comportement d'achat:
ceci symbolise à juste titre la transition d'une société de production à une société de
consommation, comme l'a caractérisée Zygmunt Bauman. $\mathrm{Au}$ cours des quarante dernières années, Spierenburg a acquis une renommée internationale grâce à ses recherches sur l'histoire du châtiment et de la violence. "L'utilité de la criminologie historique », a-t-il déclaré à l'issue de sa leçon inaugurale du 7 septembre 2007 à I'Université Erasmus de Rotterdam, «se situe non seulement dans 
l'approfondissement de la connaissance, mais aussi dans la rectification des représentations politiques concernant les arrière-plans sociaux du crime et du contrôle social. II n'y a pas de leçons simples à tirer du passé, mais une analyse historique aide à situer les problèmes contemporains dans un contexte plus large " ${ }^{1}$. Selon Spierenburg, les questions relatives à l'évolution du seuil de violence occupent une place importante du point de vue de la criminologie historique ; cela concerne aussi bien la nature du crime que la nature du contrôle social, tant sous son aspect formel (sanction par les autorités publiques) qu'informel (contrôle par les citoyens)².

C'est précisément dans ce domaine qu'il s'est spécialisé et s'est fait reconnaître Son premier livre, The Spectacle of Suffering (1984), a été particulièrement bien accueilli. Des œuvres ultérieures, telles que The Prison Experience (1991) et A History of Murder (2008) (qu'il juge personnellement meilleures que The Spectacle of Suffering), ont également été répertoriées comme des classiques en criminologie historique. En 2013 paraît, Violence and Punishment, un recueil de textes déjà publiés ou traduits en anglais, accompagnés d'un bilan personnel de son contact avec Norbert Elias, le sociologue allemand bien connu qui l'a fortement influencé. La même année, il a fait ses adieux en tant que professeur de criminologie historique à l'Université Erasmus avec un discours «Please, please me's number one $»^{3}$, dans lequel - fait remarquable pour un historien - il jette un regard sur la période récente depuis les années 1960.

Dans un département d'histoire, chacun doit s'en tenir à sa période préférée ou assignée, mais chez les criminologues, Spierenburg était l'historien, qui pouvait revendiquer l'exclusivité sur la longue histoire humaine de la violence et du châtiment : " toute l'histoire (était) pour moi», a-t-il dit dans son discours d'adieu ${ }^{4}$. C'est ainsi qu'il ne s'est que récemment aventuré sur des thèmes contemporains, tels que la punitivité croissante, la violence « gratuite » ou la montée de la figure de la victime ${ }^{5}$.

Dans les années 1960, Spierenburg était étudiant. Le thème de son discours d'adieu était-il également inspiré par la nostalgie? Était-ce un homme plus âgé qui parle ici, considérant que c'était le bon temps et qu'il n'y a plus aujourd'hui aux commandes que des bureaucrates bornés, des xénophobes et des moralistes conservateurs qui pourrissent l'atmosphère?

Non, ce n'était pas de la nostalgie - même si la musique était alors bien meilleure qu'aujourd'hui. Quand j'ai commencé à penser à mon discours d'adieu, je me suis dit : «2013, c'est cinquante ans après la percée des Beatles. Je veux en faire quelque chose ". C'était la raison première. Mais oui, en effet, j'ai vécu les années 1960 comme un grand moment et c'est ce qui s'y trouve aussi.

Nous sommes curieux de savoir quel regard Spierenburg porte sur sa vie et sa carrière. Au début de la conversation, qui a duré plus de deux heures, il nous a confié qu'il avait écrit une autobiographie : I'historien qui décrit, commente et archive son propre parcours de vie, pour la postérité. Le fait que nous n'ayons été autorisés à n'en examiner et utiliser qu'une petite partie (les années 1948-1965) pour cette contribution suggère qu'il pourrait contenir des propos fracassants. Pour l'heure, la version intégrale reste «for his eyes only» (pour ses seuls yeux).

\section{Grandir dans une famille catholique à Haarlem}

Pieter Spierenburg est né à Haarlem le 2 juin 1948 et a grandi dans un quartier ouvrier à la périphérie du centre-ville. Il le décrit comme un «environnement exclusivement catholique », mais «loin d'être strict au point d'en garder un tic » ${ }^{6}$. Le jeune Pieter Spierenburg vivait dans un «monde presque exclusivement composé de garçons » (p. 7). Dans la rue, il jouait au football, comme son contemporain Johan Cruijff, « qui jouait cent fois mieux que moi »(p.7). Dans l'autobiographie, nous lisons peu de choses frappantes dans ces premières années de vie. Spierenburg parle même de «l'ennui terrible 
des années cinquante» (p. 7). Fait remarquable, qui apparait aussi dans la conversation, son père a été admis deux fois dans une institution [psychiatrique].

Je viens [...] d'un "nid» catholique typique. Mon père a eu des problèmes psychologiques. Ils l'ont enfermé deux fois - probablement à tort - dans un asile de fous. Et à la fin on a conclu qu'il devrait travailler la terre ou quelque chose comme ça. Il combinait de façon inhabituelle un catholicisme vraiment profond empreint d'un sens du péché avec une attitude sociale progressiste. Et il n'existait probablement pas de place pour cela. Le résultat est qu'il est devenu ouvrier. Dans une lettre de mon oncle - le frère aîné de mon père - datant de 1938, on lit : " Il s'est réconcilié avec l'existence en tant qu'ouvrier». Ma mère venait d'une famille bourgeoise modeste. Elle était l'aînée de neuf enfants. Elle devait gagner de l'argent comme servante, alors qu'elle voulait étudier. Pendant la guerre, elle a encore obtenu un diplôme d'études commerciales. Malgré leur condition ouvrière, mes parents projetaient sur moi, pour ainsi dire, les ambitions intellectuelles qu'ils n'avaient pu réaliser eux-mêmes.

Sa mère souffrait d'une peur extrême de la saleté. «C'est un petit miracle que je m'en sois bien sorti », note Spierenburg dans son autobiographie (p. 1). Sa mère sera également internée pendant un certain temps, de sorte que lui et sa sœur cadette ont été placés dans un foyer pour enfants pendant environ six semaines. Spierenburg le décrit comme une «période très triste. Le plus désagréable consistait principalement dans le déclassement social que je ressentais en partie inconsciemment mais sans équivoque. J'ai fini par avoir une vie de famille, aussi déficiente soit-elle, dans un environnement d'exclus » (p. 10).

L'intérêt pour l'histoire s'est manifesté très tôt: à partir de la cinquième année primaire, lorsqu'il découvrit cette matière pour la première fois, ce fut sa matière favorite (p. 13). Ce qui explique sans doute pourquoi, malgré de bons résultats en mathématiques, en chimie et en physique, il choisirait les sciences humaines (p. 19-20). Ce choix s'est également avéré fructueux pour une autre raison: "En suivant le programme alpha (sciences humaines) facile, j'ai pu me plonger plus à fond dans la culture des jeunes, ce qui a été excellent pour mon développement personnel»(p. 24). Que Spierenburg se soit investi également dans cette culture de la jeunesse par la suite ressort à l'évidence dans un certain nombre d'éléments biographiques de son discours d'adieu :

Lorsqu'une «foire de la jeunesse " s'est tenue à Haarlem à l'été 1970 et que les organisateurs n'ont pas pu louer l'espace pour un stand, ils l'ont donné aux Kabouters (gnomes) (une réincarnation temporaire des Provos) et à Dolle Mina ${ }^{7}$. Bien sûr, les deux se sont servis de ce geste en cachette pour protester contre le cirque commercial de la foire, sous le slogan «ne vous déshabillez pas ». Le slogan était accompagné d'une photo d'un jeune homme nu et d'une jeune femme; les deux protagonistes faisaient partie du réseau des Kabouters. Les organisateurs ont retiré la photo au bout d'une journée et malheureusement les négatifs ont également été perdus. Je ne vous dirai pas qui était le jeune homme sur la photo ${ }^{8}$.

Dans son discours d'adieu, Spierenburg évoque également, bien qu'implicitement, son implication dans les actions contre la moralisation du Red Light District, qui a été considérée comme la véritable raison pour laquelle le projet 1012 (ainsi nommé d'après le code postal) de la municipalité d'Amsterdam visait à réduire «l'infrastructure criminogène » dans cette zone de prostitution et à « rétablir l'équilibre perturbé entre vie, travail et loisirs ». C'est un jargon officiel quelque peu vague, mais de nombreux criminologues avaient déjà appelé à la fermeture des lieux de prostitution, en raison de l'interdépendance croissante avec le crime organisé. Dans son discours d'adieu, Spierenburg a parlé d'un autre changement en ce qui concerne le développement de la prostitution : "Comme élément intrigant de tous les développements récents, on trouvait aussi un besoin croissant 
d'identifier des victimes partout. Récemment, ce sont les prostituées qui sont devenues la cible principale de cette victimation perçue ».

Lorsque le maire d'Amsterdam de l'époque, Job Cohen, a été confronté sur la chaîne de télévision locale AT5 d'Amsterdam à la thèse de Spierenburg selon laquelle le projet de réduire la criminalité supposément liée à la prostitution en la privant d'opportunités et en la remplaçant par des entreprises «de qualité » était irréaliste, ne contribuait pas à un environnement sûr pour les travailleuses et travailleurs du sexe et serait, au surplus, un suicide économique pour la capitale, Cohen a répondu brièvement: "Oui, mais il est professeur de criminologie historique». Spierenburg avait-il une image démodée et romantique de la prostitution et pensait-il qu'en «nettoyant» le Red Light District, les conquêtes des années 1960 seraient annulées?

Je me considère certainement comme un enfant des années 1960. Je n'aime donc pas la pudibonderie et la moralisation d'aujourd'hui, mais je ne pense pas romancer la prostitution en disant que la lutte contre les abus et la criminalisation de la prostitution en tant que telle sont deux choses très différentes. Le projet 1012 était fondé sur toutes sortes d'hypothèses non prouvées concernant la prostitution forcée et les criminels propriétaires de "vitrines ». La Cour des comptes d'Amsterdam l'a confirmé par la suite en concluant en mai dernier [2018] que le projet 1012 avait échoué. J'en déduis aussi que la municipalité n'était pas tant préoccupée par une approche efficace de la criminalité organisée, mais plutôt une fois de plus par l'expression d'un nouveau moralisme bourgeois, où il fallait se débarrasser des éléments moins « convenables » de la vie urbaine.

Cette implication et cet engagement ne sont peut-être pas surprenants à la lumière de l'atmosphère de critique du pouvoir et de l'autorité à la fin des années 1960 et dans les années 1970, mais ils ont posé un défi particulier à un historien qui suit les traces d'Elias et est très attentif à la distanciation : «Dans ces années, je faisais une distinction claire entre ma participation à la culture de la jeunesse et mes activités universitaires; entre ma vie personnelle et ma vie de chercheur débutant », rappelle-t-il dans Violence and Punishment ${ }^{\text {. }}$. Qu'il ait toujours suivi de près cette ligne de conduite ressort également de ce qu'il ait été piqué par une discussion (par Dyer) de son travail : « II ne peut pas connaître mes opinions personnelles; peut-être que je hais les gens civilisés, auquel cas mon opinion serait pessimiste $»^{10}$. Et l'une des rares critiques qu'il adresse à Twee eeuwen gevangen (1990) ${ }^{11}$ de Herman Franke, une étude éliasienne consacrée à l'histoire de l'emprisonnement aux PaysBas, était que Franke aurait exprimé trop fortement ses sentiments personnels (surtout son aversion pour l'emprisonnement cellulaire) ${ }^{12}$. Cette tension entre l'engagement et la distance a également joué un rôle dans le choix du thème de sa recherche doctorale :

J'ai moi-même vécu les années 1960 comme de superbes années. Au début, je me suis dit: "Eh bien, je vais faire ma thèse de doctorat sur le mouvement Provo ", mais quand je suis entré en contact avec Norbert Elias, j'ai pensé : «Non, ce n'est pas possible. » Dans ce cas, il y a trop d'implication. Ça peut ne pas bien se passer.

\section{Le rôle de Norbert Elias}

Spierenburg a étudié I'histoire à I'Université d'Amsterdam: "Qu'il existât une discipline comme la sociologie, je ne le savais même pas quand je suis allé étudier. Pour moi, I'histoire était la discipline sociale, celle qui était également d'actualité, qui disait quelque chose sur la société humaine ».

À l'automne 1969, il rencontrera la personne et l'œuvre de Norbert Elias, conférencier invité régulier à Amsterdam durant cette période. Dans Violence and Punishment, Spierenburg ${ }^{13}$ mentionne également l'importance d'un essai de Jan Romein, intitulé Theoretical history, qui 
mentionne explicitement le travail $d^{\prime}$ Elias $^{14}$. Comment la première rencontre avec Elias s'est-elle passée?

Presser ${ }^{15}$ était un professeur populaire, mais il était aussi un peu antithéorique, quelqu'un qui voyait l'histoire d'abord comme un récit. Par exemple, j'ai dû faire un devoir pour mon examen de candidature. J'avais entendu d'un professeur de lycée que la puberté avait été décrite pour la première fois au XVIII ${ }^{\mathrm{e}}$ siècle comme une période qui durerait six mois. Et qu'elle est devenue de plus en plus longue. Je m'en étais souvenu. J'ai dit à Presser : "J'aimerais écrire un article là-dessus. » Et il répondit: «Oh, non. C'est de la psychologie, et vous allez produire des affirmations que vous ne pouvez pas prouver. » Presser est devenu émérite à 70 ans. Il était le seul professeur d'histoire moderne. Et aucun successeur n'avait encore été nommé [....]. Pour un séminaire, vous pouviez alors choisir un professeur invité, Norbert Elias ». [...] Je suis allé chez Duijker ${ }^{16}$ pour la psychologie sociale et il m'a dit de ne pas prendre de cours avec lui, mais de passer un examen sur quatre gros livres, dont Über den Prozess der Zivilisation par Elias. Il ne savait pas du tout qu'Elias était à Amsterdam. [...] Et Duijker a dit à propos de ce livre : «Si vous prenez ma discipline sous l'angle historique, alors je pense que c'est la bonne manière. Je ne prescrirais jamais le livre à mes élèves, parce que c'est trop loin de leur univers. Mais vous êtes historien, alors vous pouvez le prendre pour mon examen». C'est là que j'ai commencé à le lire. Ainsi, parallèlement au séminaire, j'ai pu lire son livre.

Spierenburg devint ainsi l'un des premiers historiens à s'inspirer de la théorie de la civilisation d'Elias - à la fin de son discours, il l'appelle "mon maître ${ }^{17}$. Dans son œuvre bien connue, Elias a étudié les codes de comportement du XVe au XVIII siècle. De cette façon, il a montré que toutes sortes d'actions et de manières - se moucher, faire ses affaires, faire l'amour, etc. - commençaient peu à peu à susciter des sentiments de honte et devenaient le sujet de régulations. Dans un passage caractéristique de son livre, Elias $\operatorname{dit}^{18}$ :

Disséquer des animaux faisait [...] autrefois partie de la vie sociale de la classe supérieure. Peu à peu, on trouve de plus en plus douloureux de le voir. L'acte de trancher lui-même ne disparaît pas, car l'animal doit être coupé en morceaux si l'on veut le manger. Mais ce qui est devenu douloureux est rejeté dans les coulisses de la vie sociale. Les spécialistes le livrent en magasin ou en cuisine. Il apparaît de façon répétée que cette séparation, cette "mise en coulisse" de ce qui est devenu douloureux, est caractéristique du processus de civilisation.

La violence est également apprivoisée de cette manière : les comportements agressifs font l'objet d'une régulation et disparaissent de plus en plus de la vie publique. Une évolution similaire s'est produite en ce qui concerne le châtiment: des condamnations spectaculaires et très fréquentées sur l'échafaud ont cédé la place au châtiment sans effusion de sang et caché derrière les hauts murs de la prison. Tout comme la dissection de l'animal, la violence interpersonnelle et l'exécution du châtiment disparaissent dans les coulisses de la société humaine.

Spierenburg a consacré la majeure partie de sa vie professionnelle à l'étude de ces processus. Pourquoi ce choix?

C'est l'une des rares contributions créatives de mon directeur de thèse, M.C. Brands ${ }^{19}$. J'avais fait ma thèse de master ${ }^{20}$ sur le processus de civilisation aux Pays-Bas. Il s'agissait en fait d'un vaste test des théories d'Elias sur la base de documents en néerlandais. [...] Je n'avais rien trouvé sur l'agressivité, qu'Elias appelle Angriffslust, ou si peu. Mon idée était donc de continuer dans cette voie pour ma thèse. Et puis, à un certain moment, Brands a dit: «Pourquoi ne pas consacrer une étude au 
châtiment? Eh bien, dans le passé, il y avait beaucoup de gifles et à partir d'un certain moment, plus. Pourquoi ne pas vous concentrer là-dessus? "Sur le plan international, il existait quelques livres sur la criminalité sous l'ancien régime et aux Pays-Bas, il semblait y avoir un groupe qui s'intéressait à ce sujet. J'ai donc assez tôt pris le train de l'histoire de la criminalité et de la justice pénale qui démarrait - et je m'y suis toujours accroché.

Dans Violence and Punishment, il évoque également le lien entre le thème de sa thèse - la disparition des châtiments corporels publics - et la théorie de la civilisation, un lien évident:

Il n'était pas nécessaire d'être un spécialiste pour savoir que les marques au fer rouge et les pendaisons publiques étaient autrefois communes et acceptées et qu'elles ne le sont plus aujourd'hui. Évidemment, cela pourrait être analysé en termes de processus civilisateurs. Il s'est avéré qu'il y avait beaucoup de livres sur les exécutions et la torture dans le passé, mais ils étaient tous purement descriptifs, ou, pire, écrits dans une perspective d'applaudissement du progrès humain. L'ouvrage de Foucault sur le sujet n'est apparu que quelques années plus tard. C'était donc un défi d'écrire sur les changements dans les châtiments dans le langage de la sociologie historique ${ }^{21}$.

Ici apparaît également l'intérêt pour la théorie : la recherche historique ne se limite pas à la collecte de faits qui sont mis à l'épreuve. L'étude de la violence et du châtiment fournit une clé qui ouvre la porte à une meilleure compréhension des sociétés passées et présentes. Dans l'introduction de A History of Murder, Spierenburg écrit : "Le meurtre affecte toujours les valeurs fondamentales de ceux qui participent à l'acte et en sont témoins, fournissant ainsi des informations précieuses sur la culture, la hiérarchie sociale et les relations de genre $» 22^{22}$ Dans un essai de 2004, il fait une remarque similaire au sujet de la punition: «L'objectif est d'explorer de quelle manière les changements dans la punition reflètent des développements plus larges et à long terme dans la société ; d'apprendre, à travers l'étude de la punition, comment ces développements sont interdépendants; pour savoir si tout cela peut améliorer notre compréhension de la structure de notre propre société et de nous-mêmes $»^{23}$

Mais qu'en est-il des autres théories ? L'année de sa rencontre avec Elias, en 1969, a eu lieu un an après la réédition de l'ouvrage pénologique classique Punishment and Social Structure de Georg Rusche et Otto Kirchheimer, un livre publié à l'origine en 1939, tout comme le grand livre d'Elias. Quelle était sa position à ce sujet?

J'ai aimé, mais j'étais un peu déçu. Et pourquoi était-ce une déception? Parce que c'est Norbert Elias qui me l'avait recommandé. Quand je lui ai dit pour la première fois que j'allais me concentrer sur la punition, il m'a dit: «Eh bien, je connais un livre, de nature théorique et qui en parle. J'ai connu les deux auteurs. Un livre de Rusche et Kirchheimer » [...] Et ensuite j'en ai parlé avec lui et je lui ai dit que c'était très marxiste. «Oui, oui, c'est vrai, mais ce sont les seuls qui en parlent un peu théoriquement. » Alors, c'est comme ça. Mais à un certain moment, j'ai développé ma propre théorie.

En tout état de cause, Spierenburg s'est avéré avoir peu d'affınités avec les sociologues marxistes:

Beaucoup d'étudiants parmi les plus sympathiques à Elias voulaient aborder les grandes questions, comme le font les marxistes, mais ils n'aimaient pas le marxisme lui-même. Et ce fut aussi mon cas. Je pense aussi à un incident dans le jardin du séminaire historique [...]. Les étudiants marxistes, presque sans exception, venaient de milieux aisés. À l'époque, il existait une émission populaire appelée Hadimassa ${ }^{24}$, dans laquelle Ton van Duinhoven persiflait à ce propos: dans une réunion de 
plusieurs hommes d'affaires, l'un dit: "Oui, mon fils est communiste de consei ${ }^{25}$ » et un autre "Ah Oui ? Oh, le mien est anarchiste!» Je savais pertinemment que ces gens, qui parlaient tant des travailleurs, ne connaissaient pas du tout la classe ouvrière. Et je connaissais fichtrement bien cette classe ouvrière : j'y ai grandi. À un moment donné, il existait un groupe de travail sur Histomat [ndr: matérialisme historique], qui continuait à débattre jusque tard dans le beau et grand jardin de l'Institut. Et j'étais toujours seul dans le jardin. Ensuite, sont arrivés des jardiniers qui devaient arroser le jardin ou quelque chose comme ça. Les membres d'Histomat ont levé les yeux très troublés par les vrais travailleurs qui sont arrivés et estimaient que les chaises devaient être enlevées, et ils sont rentrés. Puis j'ai aidé ces gens en rangeant les chaises dans l'abri de jardin. Quoi qu'il en soit, c'est un événement symbolique pour moi : ces marxistes, qui viennent de milieux riches, ont la bouche pleine d'ouvriers, mais ils ne savent rien d'eux. C'est l'une des raisons pour lesquelles j'avais auparavant choisi Elias.

\section{Eliasiens et Foucaldiens}

Son choix pour Elias était-il aussi un choix contre Michel Foucault qui, au milieu des années 1970, bénéficiait d'un grand intérêt pour son étude Surveiller et punir? En 1978, dans une publication sur la fonction sociale des exécutions pénales publiques, Spierenburg formulait deux critiques à l'égard du philosophe français. D'une part, Foucault ne prêterait aucune attention au processus : il n'y a pratiquement pas de préhistoire de la prison chez Foucault et les châtiments publics disparaissent très abruptement de la scène. D'autre part, Spierenburg estime que Foucault n'a pas vraiment de théorie: "Sa description méticuleuse s'accompagne d'un minimum d'explications, même lorsqu'il place l'émergence du système carcéral dans un cadre plus large de discipline croissante dans toutes sortes de domaines. La discipline n'est pas liée à d'autres développements sociaux. II nous reste une montagne de rhétorique qui n'augmente certainement pas la clarté de l'argument ${ }^{26}$. Dans The Spectacle of Suffering, Spierenburg va plus loin: il y présente la théorie de la civilisation d'Elias comme un « contre-paradigme » à la vision foucaldienne du châtiment ${ }^{27}$.

Surveiller et punir a été publié en 1975. La traduction anglaise a été publiée en 1977. Ce n'est qu'alors que le livre est devenu connu à l'étranger. J'ai obtenu mon doctorat en 1978, et donc j'avais lu le livre. J'y fais également référence de temps à autre et j'ai mentionné quelques points critiques. Par exemple, Foucault croit que le désordre autour de l'échafaud n'est apparu que vers la fin de l'Ancien Régime. Cependant, j'avais des données empiriques prouvant que la plupart des spectateurs était normalement intéressés au spectacle, sauf lorsque des insurgés ou des grévistes - des gens auxquels les spectateurs pouvaient s'identifier - étaient punis. Vous pouvez déjà le voir en 1650. C'était ma principale critique. Mais je me suis aussi rendu compte que la vision de Foucault était en fait une antithèse de l'approche d'Elias, qui se concentre sur le changement à long terme, alors que Foucault parle d'un revirement total en quatre-vingts ans - en fait, malgré ce qu'il a écrit dans l'Histoire de la folie, où ces maisons disciplinaires sont mentionnées. Il aurait donc dû savoir qu'il s'agissait d'un développement à plus long terme. Il a ses adversaires parmi les historiens, mais ce sont souvent ces empiristes, qui vérifient tout, mais qui n'aiment pas la théorie. Ce n'est pas de cela qu'il s'agissait pour moi : j'aime la théorie, mais j'aime les bonnes théories. Alors je me suis dit: «Eh bien, ce serait peut-être une bonne publicité si je proposais explicitement le livre comme une approche alternative, contre l'approche 
de Foucault ». Et c'est ce que j'ai fait. Et à ma demie surprise, il a été énormément repris.

C'est cette représentation quelque peu provocatrice en termes de paradigme et de contreparadigme qui lui a mis le vent en poupe. Dans un compte-rendu, David Garland (1986) a longuement traité de The Spectacle of Suffering, soulignant l'importance du livre pour la sociologie de la peine, notant que du point de vue d'une sociologie criminelle multidimensionnelle il est préférable de ne pas parler en termes de paradigmes : « II n'est pas question de "paradigme", de "contre-paradigme" et d'un choix entre eux [...] l'objectif devrait être d'amener ces études distinctes, qui sont différemment ciblées mais loin d'être incommensurables, dans une relation complémentaire où chacune éclaire l'autre ${ }^{28}$.

Il est frappant de constater que Spierenburg est devenu plus modéré envers Foucault au fil du temps. Dans un article de 2004, il relit Foucault et semble suggérer de porter un regard critique sur les « Foucaldiens » plutôt que sur Foucault lui-même. Pourquoi en est-il ainsi ?

En 1984, dans The Spectacle of Suffering, j'étais quelque peu exaspéré et cela ne m'a pas fait de mal. Cela a également contribué à ma reconnaissance - si je puis me permettre de m'exprimer ainsi - en tant que scientifique. La raison pour laquelle j'y suis revenu était une invitation de criminologues d'Oslo à donner une conférence dans laquelle ils m'ont écrit : «Eh bien, peut-être pouvez-vous nous parler de l'approche d'Elias et de la critique de Foucault ». Oui, je peux le faire. Et quand ai-je lu Foucault ? Eh bien en 1975, lors de la parution du livre, mais jamais par la suite. Alors je l'ai relu, et il n'y a finalement pas un mot impertinent dans le livre. En fin de compte, je maintiens deux points de critique : il ne s'agit pas d'un changement rapide en quatre-vingts ans, mais d'un développement à long terme; et je reste critique quant à la manière dont il utilise le concept de « pouvoir ».

\title{
Les criminologues historiens d'Amsterdam qui se nommaient souvent Herman
}

\begin{abstract}
Aux Pays-Bas, un paysage criminologique historique était en train d'émerger dans lequel les travaux de Michel Foucault et de Norbert Elias, ainsi que des travaux plus empiriques et historiques, jouaient un rôle. "Et ils semblaient tous s'appeler Herman », dit Spierenburg: les deux empiricistes Herman Diederiks et Herman Roodenburg, ainsi que l'Eliasien Herman Franke et le pseudo-Foucaldien Herman Bianchi ont joué un rôle important dans la création d'une communauté criminologique historique aux Pays-Bas. Comment voit-il l'approche athéorique de la criminologie historique?
\end{abstract}

Bianchi était un exemple typique de quelqu'un qui préfère abuser de l'histoire en faveur de ses propres conceptions politiques. Ces dernières ont coloré son image de l'histoire. Et ce n'était pas mon approche, bien sûr. Mais il en savait beaucoup. Aussi sur l'histoire, même s'il n'était pas historien. Je l'ai rencontré en 1974. Au cours de l'été ou de l'automne de la même année, j'ai assisté à la première réunion du groupe de travail sur l'histoire du droit pénal, qui avait été fondé par Herman Diederiks et Sjoerd Faber. Et Herman Bianchi participait à cette réunion ${ }^{29}$.

Malgré les objections idéologiques à une grande partie de son travail, Spierenburg a également été en mesure d'apprécier Bianchi.

J'ai quand même défendu Herman Bianchi. Une fois, je suis allé chez Joop Goudsblom ${ }^{30}$ avec lui et Herman Roodenburg. Roodenburg venait d'obtenir son diplôme et il allait faire son doctorat chez Goudsblom. Et je pense qu'il voulait qu'Herman Bianchi soit co-directeur en raison du thème criminologique de ses recherches doctorales. Mais Joop n'aimait pas ça du tout. Et je l'ai accompagné en 
tant que médiateur. Puis j'ai dit à Joop : « Oui, tout le monde n'a pas exactement votre opinion sur ce domaine. Vous avez dû travailler avec des collègues qui n'avaient pas exactement vos propres opinions. » [...] J'ai aussi dit : « Il est très érudit. » Joop ne le pensait pas. Il ne l'aimait pas du tout et il s'y est tenu. Mais la fin de l'histoire, c'est que Bianchi a trouvé de l'argent pour Roodenburg et est devenu son directeur de thèse.

Herman Franke était à côté de Spierenburg, l'« Eliasien » dans le domaine de l'histoire du droit pénal. Pourtant, il y eut relativement peu de contacts entre les deux.

On n'a jamais vraiment travaillé ensemble, non. Oui, un peu, quand il a commencé avec l'histoire des crimes de sang, quand je travaillais aussi là-dessus. Je lui ai parlé à un moment ou à un autre, parce qu'il avait des chiffres sur l'Amsterdam d'aujourd'hui. J'ai commencé à l'époque où la police elle-même commençait à compiler systématiquement les chiffres des crimes de sang à Amsterdam [...]. Donc, j'avais rédigé un court passage dans mon premier article sur les crimes de sang à Amsterdam à partir des données de l'époque actuelle. C'était une référence à ses conclusions. Nous étions d'accord sur ce point de temps en temps, mais nous n'avons pas vraiment travaillé ensemble. Peu de temps après, il est aussi devenu romancier : il a dit adieu à la criminologie.

Observons également que Spierenburg n'entrera dans les cercles criminologiques qu'à un stade ultérieur, alors que le sociologue Franke a été le premier à s'y impliquer. N'était-ce pas principalement ces mouvements contraires disciplinaires qui ont conduit à si peu de coopération entre les deux éliasiens ? Cela a dû être en partie le cas, mais cela n'explique pas les critiques mutuelles. Qu'est-ce que tu en penses?

Eh bien, je ne sais pas. Nous avons eu quelques frictions au début. En fait, il ne pensait pas que mon approche était assez éliasienne; il l'a même appelée structurofonctionnaliste, ce qui était la plus grosse injure en sciences sociales à l'époque. J'ai trouvé cela drôle en soi, car avec mes collègues historiens de Rotterdam, je l'ai utilisé à mon avantage. "Vous dites toujours que je suis trop occupé avec Elias, mais un autre éliasien m'accuse de structuro-fonctionnalisme ». J'ai parfois eu l'impression qu'Herman Franke pensait qu'il devait être le grand artisan de l'application de la perspective historique d'Elias au développement de la punition et que c'était aussi le contexte de ses attaques théoriques contre moi. Après, on a été plus amicaux l'un avec l'autre, tu sais.

\section{Le rôle de l'école d'Amsterdam}

Quand bien même il est inspiré par Elias, Spierenburg a eu très peu de contact avec lui tout en écrivant sa thèse. Dans Violence and Punishment Spierenburg écrit qu'à la fin, quand sa thèse était presque terminée, il en a brièvement discuté avec Elias. Et c'était tout?

J'ai en effet écrit ma thèse principalement de mon propre chef. Je n'en parlais qu'une fois de temps en temps avec Elias. À un moment donné, il est aussi parti pour Bielefeld. Je ne me souviens pas exactement quand, mais pendant un certain nombre d'années, il n'a guère été présent à Amsterdam non plus. Cas Wouters ${ }^{31}$ m'a dit un jour qu'il avait parlé à Elias et qu'ils avaient parlé de moi pendant un certain temps. Et puis Norbert avait aussi dit : «Oui, cette thèse est tout à fait son propre ouvrage, sur la répression et son histoire. » Et pour Cas c'était un compliment. [....] La dernière fois que j'ai vu Elias, c'était à son $90^{\mathrm{e}}$ anniversaire. Ce fut une grande rencontre dans 
l'église luthérienne sur le Spui, l'auditorium de l'Université d'Amsterdam. Et c'était en 1987.

Dans quelle mesure Spierenburg était-il intégré à l'école d'Amsterdam ? Avait-il des pairs qu'il fréquentait? Le contact avec Anton Blok, par exemple, aurait été évident, car son travail se situe également à l'interface des sciences sociales et de l'histoire, et il a également écrit sur des thèmes criminologiques. Là aussi, on retrouve peu de traces.

J'ai eu des contacts avec Anton Blok, mais ça a pris une autre direction. J'ai fait la connaissance d'Anton en 1974 dans le groupe de travail d'histoire du droit pénal. Et il s'est avéré qu'il avait lu ma thèse de master. Au sein de ce groupe de travail, il y avait aussi un groupe théorique. Il comprenait Anton Blok et moi, Herman Bianchi, Herman Diederiks, Sjoerd Faber et Arend Huussen. Et cela a finalement abouti à la création d'une communauté internationale pour la criminologie historique. À l'origine, Herman Diederiks avait pris l'initiative d'organiser une conférence internationale. En Angleterre, en Belgique et en France, certaines personnes travaillent déjà dans ce domaine. Dans la communauté des criminologues historiques, nous étions maintenant connus sous le nom de «Groupe néerlandais ». Cela vient de ce que j'ai écrit dans des lettres au sujet du congrès à organiser, que j'étais le Secrétaire international du "groupe néerlandais». En 1978, le grand Congrès international quadriennal d'histoire sociale a eu lieu à Édimbourg, où nous avons fondé l'International Association for the History of Crime and Criminal Justice ${ }^{32}$.

II est quelque peu surprenant que quelqu'un qui est en tout cas dans la tradition de l'école d'Elias à Amsterdam aille au Centre d'histoire sociale de l'Université Erasmus de Rotterdam dès avant la soutenance de son doctorat. Comment cela s'est-il produit?

Ce qu'on appelle aujourd'hui l'école d'Amsterdam n'existait pas du tout à l'époque. Et, regardez, j'étais historien. Donc ils ne m'auraient pas si facilement embauché, ils auraient d'abord embauché des sociologues. Donc faire quelque chose là-bas, en sociologie, n'était pas évident à l'époque. Parce que, bien qu'Elias fût bien sûr très interdisciplinaire, il existait des distinctions claires entre ces disciplines. Mais j'étais au chômage à l'époque et j'ai postulé à tous les postes universitaires dont je pensais «bon, je peux y prétendre ». Lorsqu'une faculté innovante a été créée à l'université Erasmus, vraiment centrée sur l'histoire de la société, j'ai pensé que j'aurais de bonnes chances d'y réussir. Et à la fin, j'ai été accepté là-bas aussi. C'est aussi simple que ça.

\section{Histoire sociale et criminologie à l'Université Erasmus de Rotterdam}

Bien que Pieter Spierenburg soit associé depuis 1977 au Centre d'histoire sociale de I'Université Erasmus de Rotterdam (aujourd'hui section de l'École Erasmus d'histoire, culture et communication), il semble avoir eu relativement peu de liens avec ses collègues. II est finalement devenu professeur à l'École de droit Erasmus, dans le département de criminologie. Bien que Spierenburg soit l'un des auteurs les plus éminents des deux facultés au niveau international, il n'est devenu professeur que tardivement. Comment l'explique-t-il ?

La raison la plus importante pour laquelle je ne suis jamais devenu professeur d'histoire sociale est simplement Siep Stuurman ${ }^{33}$ : c'était l'homme le plus important à l'époque. Plusieurs autres collègues influents ne pensaient pas non plus qu'il était nécessaire que je devienne professeur. Je n'ai jamais bien compris pourquoi. J'ai 
souvent été contre la nomination d'historiens de troisième ordre comme professeurs, alors que des candidats internes bien meilleurs ont été écartés. Ils m'en ont tenu rigueur. [...] Mais j'étais un bon ami de la moitié de cette section d'histoire, et il y en a avec lesquels j'ai encore des contacts, comme Rudolf Dekker et Paul Schulten. Dans les années 1980, mon collègue Kris Vos disait déjà : "Quels beaux livres tu écris, Pieter. Il est temps pour toi de devenir professeur. » Mais on n'en est jamais arrivé là. L'historien économique Peter Klein a toujours apprécié mon travail. Peu de temps après mon entrée en fonction, il s'est assuré que mon article De sociale functie van openbare strafvoltrekkingen [La fonction sociale de l'exécution publique des peines] soit inclus dans la série de la faculté et a fait allusion au fait que le poste de maître de conférences (à l'époque une sorte de semi professeur) pourrait m'être réservé dans quelques années. Mais bon, il est parti à Leyde. À ma grande surprise, il était présent à ma leçon inaugurale en 2007.

La pensée : « Je vais en Angleterre ou en Amérique »n'a-t-elle jamais traversé l'esprit?

Je n'ai jamais vraiment voulu partir à l'étranger. Ma maison et ma vie à Amsterdam étaient trop agréables pour ça. Il m'est bien arrivé d'y songer. Ma première chaire de professeur invité remonte à 2001 à Pittsburgh. C'était un vrai soulagement. Parce que j'y ai été traité avec respect, comme un historien important. Je me suis dis : «C'est donc possible! » Mais à la fin, rien n'en est sorti. J'ai aussi bien aimé Pittsburgh. Mais je me suis dit: « Je vais poursuivre mes contacts internationaux et mes livres sont lus dans le monde entier et c'est le plus important ».

Dans son discours d'adieu, Spierenburg ${ }^{34}$ écrit : "Pendant des années, je me suis surtout concentré sur les cercles scientifiques internationaux et c'est peut-être pour cela qu'à un moment donné, j'ai connu un décalage entre la réception de mon travail dans le monde et mon rang officiel dans le pays où je vis ». N'était-ce pas frustrant?

Oui, bien sûr que c'est vrai. J'ai parfois pensé : «Je vais devoir prendre ma retraite et je n'aurai même pas été professeur. Cela ne devrait sûrement pas être possible.» C'est principalement grâce à Henk van de Bunt et à toi (RvS) que ça a marché, en fin de compte. À mon avis, Siep Stuurman a dû en avoir quelques grincements de dents à l'époque. La criminologie était une section très jeune et ils organisaient des voyages et autres, et je m'y sentais bien.

\section{Norbert Elias et d'autres personnages importants}

En tant que membre du comité de rédaction de la revue Tijdschrift over Cultuur and Criminaliteit, Pieter Spierenburg a souvent dit qu'il était temps de se consacrer un peu plus à Norbert Elias. Pourquoi cela ? Quelle est, selon Spierenburg, la pertinence de la théorie de la civilisation d'Elias à une époque où beaucoup de gens croient que les manières redeviennent rudes et que les populistes tiennent la politique en otage? Dans l'œuvre d'Elias, la formation de l'État est l'un des éléments de la civilisation, mais depuis les années 1990 néolibérales, de nombreuses tâches étatiques ont été privatisées. Et en plus de la «dés-étatisation», de nombreuses relations supranationales ont vu le jour. La décivilisation est-elle en cours?

L'émergence de relations supranationales signifie en fait que la formation de l'État se poursuit à un niveau supérieur. Je suis très pro-Européen. Je sais que si ces antiEuropéens obtiennent ce qu'ils veulent, les multinationales se mettront à leur place, parce qu'elles seront aux commandes. Les États européens actuels ne peuvent pas du tout concurrencer les multinationales. Cela n'est possible que dans une Union 
européenne forte. Les populistes d'aujourd'hui s'y opposent avec véhémence et leur appel à la préservation de "notre » civilisation (dite judéo-chrétienne) est très mince, mais ma perspective historique de longue durée est encore trop ancrée pour que je puisse parler de décivilisation à ce stade.

À la fin de cet entretien, nous posons la question suivante : quelle est la personnalité la plus significative pour vous. «Et tu ne peux pas dire Elias ». Pieter Spierenburg se tait un instant et réfléchit profondément. II finit par mentionner I'historien français Yves Castan, qu'il connaît entre autres à travers le comité de rédaction de la revue Crime, Histoire \& Sociétés ${ }^{35}$. Mais la vraie réponse n'arrive qu'un jour plus tard, par courrier électronique.

C'était un entretien agréable hier. Seule la question d'un être cher après Norbert Elias m'a un peu surpris. En y réfléchissant davantage, j'aurais bien sûr dû d'abord nommer William $\mathrm{H}$. McNeill ${ }^{36}$ : un collègue qui a aussi une perspective globale et à long terme. C'est surtout son livre Le temps de la peste de 1977 qui m'a fait une grande impression ${ }^{37}$.

\section{BIBLIOGRAPHIE}

http://www.pieterspierenburg.com/

Daems, T., De bestraffingssociologie van David W. Garland [La sociologie punitive de David. W. Garland], Den Haag, Boom Juridische uitgevers, 2009.

Elias, N., Het civilisatieproces. Sociogenetische en psychogenetische onderzoekingen [Sur le processus de civilisation. Recherches sociogénétiques et psycho génétiques], Utrecht-Antwerpen, Het Spectrum, 1982 [1939].

Faber, S., Herman Diederiks (1937-1995) en de Werkgroep Strafrechtsgeschiedenis (1973-1998). Pro Memorie, 2000, 2, 2, 384-385.

Garland, D., The punitive mentality: Its socio-historic development and decline, Contemporary Crises, 1986, 11, 2, 198-201.

Michael, W.F., Proceedings of the Seventh International Economic History Association conference, 2, vol., Edinburgh, Edinburgh University Press, 1978.

McNeill, W.H., Plagues and Peoples [Le temps de la peste, essai sur les épidémies dans l'histoire], New York, Anchor Press, 1977 [Paris, Hachette, 1978].

Romein, J., Theoretical history, Journal of the History of Ideas, 1948, 9, 1, 53-64.

Rousseaux, X., Crime, Justice and Society in Medieval and Early Modern Times: Thirty Years of Crime and Criminal Justice History, Crime, History \& Societies, 1997, 1, 1.

Spierenburg, P., De sociale functie van openbare strafvoltrekkingen [La fonction sociale des exécutions publiques], Centrum voor Maatschappijgeschiedenis, Mededelingen no. 4, Rotterdam, Erasmus Universiteit Rotterdam, 1978.

Spierenburg, P., The Spectacle of Suffering, Cambridge, Cambridge University Press, 1984. 
Spierenburg, P., Elias and the History of Crime and Criminal Justice: A Brief Evaluation, IAHCCJ Bulletin, 1995, 17-30.

Spierenburg, P., Punishment, power and history: Foucault and Elias, Social Science History, 2004, $28,4,607-636$.

Spierenburg, P., Apegatjes achtervolgers. Sociale controle tussen verschuivende geweldsdrempels en veranderende noties van privacy [Le contrôle social entre les déplacements des seuils de violence et l'évolution des conceptions de la vie privée], in Van De Bunt, H., Spierenburg, P., van Swaaningen, R., (Eds.), Drie perspectieven op sociale controle [Trois perspectives sur le controle social], Den Haag, Boom Juridische uitgevers, 2007, 67-112.

Spierenburg, P., A History of Murder, Cambridge, Polity, 2008.

Spierenburg, P. Violence and Punishment, Cambridge, Polity, 2013a.

Spierenburg, P., Please, please me's number one. Maatschappelijke veranderingen sinds de jaren zestig en hun weerslag op het beeld van misdadigers en slachtoffers [Les changements de société depuis les années soixante et leur impact sur l'image des criminels et des victimes], 2013b, Den Haag, Boom Lemma.

Spierenburg, P., Autobiografie van Pieter Spierenburg (periode: 1948-1965). Ongepubliceerd [Autobiographie de Pieter Spierenburg (période 1948-1965), inédit], 2018, 26 p.

\section{NOTES}

1. Spierenburg (2007, p. 102).

2. Spierenburg (2007, p. 69).

3. La citation vient de la chanson Nothing has been proven des Pet Shop Boys, écrite pour le film Scandal en 1989. Le film évoque le scandale sexuel et politique John Profumo/Christine Keeler de mars 1963. Elle fait référence au titre de la première chanson à succès des Beatles Please, Please Me...) sortie au moment où éclate le scandale et numéro 1 des ventes en Angleterre pendant la durée du scandale de mars à juillet 1963. (Thompson, Gordon, Please, Please Me : Sixties British Pop, Inside Out, Oxford UP, 2008. https://en.wikipedia.org/wiki/Nothing_Has_Been_Proved) [Ndt].

4. Spierenburg (2013b, p. 7).

5. Voir par exemple Spierenburg $(2008,2013 a, 2013 b)$.

6. Spierenburg (2018, p. 4).

7. Kabouters : parti politique anarchiste néerlandais, actif de 1969 à 1974, issu du mouvement Provo: mouvement libertaire actif dans les années 1965 et 1970 aux Pays-Bas. Dolle Mina's: groupe féministe issu de la jeunesse socialiste néerlandaise, actif dans les luttes contre la discrimination dans les années 1970 [Ndt].

8. Spierenburg (2013b, p. 24-25).

9. Spierenburg (2013a, p. 177).

10. Spierenburg (1995, p. 21).

11. Herman Franke (1948-2010), journaliste, sociologue, criminologue, romancier et essayiste, auteur de Twee eeuwen gevangen. Misdaad en straf in Nederland [Deux siècles de prisons. Délinquance et peine aux Pays-Bas], 1990, traduit en version courte The Emancipation of Prisoners. A Socio-Historical Analysis of the Dutch prison Experience, 1995. Il est notamment connu pour son roman De Verbeelding, 1998.

12. Spierenburg (1992).

13. Spierenburg (2013a, p. 179).

14. Voir Romein (1948, p. 62). 
15. Jacob Presser (1899-1970) professeur d'histoire politique à l'Université d'Amsterdam jusqu'en 1969 http://resources.huygens.knaw.nl/bwn1880-2000/lemmata/b/bwn4/bwn2/bwn1/presser [Ndt].

16. Hubertus (Bert) Carl Johannes Duijker $(1912,1983)$, professeur de pychologie à l'Université d'Amsterdam [Ndt].

17. Spierenburg (2007, p. 103).

18. Elias, 1982 [1939], Part 1, p. 170-171.

19. Maarten Cornelis Brandts (1933-2018), professeur d'histoire nationale à l'Université d'Amsterdam [Ndt].

20. Avant l'organisation en bachelor/master, aux Pays-Bas la doctoraal scriptie correspondait au mémoire de master, ouvrant la porte au doctorat [Ndt].

21. Spierenburg (2013a, p. 179).

22. Spierenburg (2008, p. 1).

23. Spierenburg (2004, p. 625).

24. Émission populaire de la télévision néerlandaise publique VARA (Association des travailleurs radio amateurs, liée au pilier socialiste et social-démocrate [Ndt].

25. Communiste de conseil, ou conseilliste, privilégiant la direction de la société par des soviets, tendance marxiste anti-léniniste par opposition au communisme de parti [Ndt].

26. Spierenburg (1978, p. 4).

27. Spierenburg (1984, p. ix).

28. Garland (1986, p. 315) ; voir également Daems (2009, p. 51-52) pour une discussion.

29. Le groupe de travail («werkgroep») strafechtsgeschiedenis a existé de 1973 à la fin des années 1990. Voir Faber (2000).

30. Johan (Joop) Goudsblom, professeur de sociologie à l'Université d'Amsterdam, introducteur et ami de Norbert Elias.

31. Casparus (Cas) Wouters, chercheur en sociologie à l'Université d'Utrecht, disciple d'Elias et de Joop Goudsblom, auteur de Sex and Manners: Female Emancipation in the West 1890-2000, London, Sage, 2004.

32. Association internationale pour l'histoire du crime et de la justice pénale. Les deux premières réunions du Nederlandse Werkgroep Strafrechtsgeschiedenis furent organisée en 1977 à Amsterdam et Leiden. L'International Association for the History of Crime and Criminal Justice (IAHCCJ) est née lors d'une session du $7^{\mathrm{e}}$ Congrès d'histoire économique (voir Michael, 1978; Rousseaux, 1997).

33. Sijbrand (Siep) Stuurman, 1946-, professeur d'histoire des idées à l'Université d'Utrecht.

34. Spierenburg (2013b, p. 40).

35. Yves Castan (1924-), professeur d'histoire moderne à l'Université de Toulouse, co-fondateur de la revue Crime, Histoire et Sociétés et auteur de Honnêteté et relations sociales en Languedoc 1715-1780, Paris, 1974.

36. William H. McNeill (1917-2016), professeur d'histoire globale à l'Université de Chicago, auteur de The Rise of the West: A History of the Human Community, Chicago, University of Chicago Press 37. William H. McNeill (1977). 


\section{AUTEURS}

\section{PIETER SPIERENBURG}

Erasmus University Rotterdam

\section{TOM DAEMS}

Leuven Institute of Criminology (LINC)

RENÉ VAN SWAANINGEN

Erasmus School of Law 